\title{
Fisioterapia en parálisis cerebral infantil discinética de tipo distónica: Estudio de un caso
}

\author{
Physiotherapy in dystonic dyskinetic infantile cerebral palsy: A case study \\ Mario I Ortiz ${ }^{a}$, Marlenne Yulieth Martínez-Cisneros ${ }^{b}$, Sandra Kristal Cortés-Márquez ${ }^{c}$
}

\begin{abstract}
:
Introduction: Infantile cerebral palsy is a neurological disorder that affects motor function, as well as muscle control, coordination and movement. The objective of this study is to describe the motor development of a child with dystonic-type dyskinetic cerebral palsy using the integral physiotherapeutic approach. Case Description: A 2-year-old boy with dystonic-type dyskinetic cerebral palsy with prematurity is presented. Intervention: Using the Gesell Scale, an initial evaluation was performed. Specific physiotherapeutic treatment was applied twice a week for 3 months. It acts on their motor, psychosocial, language and adaptive development. In the end, development was re-evaluated on the same scale. Results and discussion: The age limit obtained in the baseline evaluation was 7 months old. After the intervention, an improvement in his postural control, in his postural and balance reactions could be observed. The evolution of the child after the physiotherapeutic treatment was favorable in the period evaluated, according to the results obtained. The final evaluation with the Gesell Scale gave an age limit of 1 year 4 months. Conclusion: The short physiotherapy program applied to the patient showed favorable effects on his psychomotor development.
\end{abstract}

\section{Keywords:}

Infantile cerebral palsy, Dyskinetic, postural control, physiotherapy.

\section{Resumen:}

Introducción: La parálisis cerebral infantil es un trastorno neurológico que afecta a la función motora, así como el control muscular, la coordinación y el movimiento. El objetivo de este estudio es describir el desarrollo motor de un niño con parálisis cerebral discinética de tipo distónica mediante el abordaje fisioterapéutico integral. Descripción del Caso: Se presenta un niño de 2 años con parálisis cerebral discinética de tipo distónica con prematurez. Intervención: Utilizando la Escala de Gesell, se realizó una evaluación inicial. Se aplicó un tratamiento fisioterapéutico específico de 2 veces por semana durante 3 meses. Se actúa sobre su desarrollo motriz, psicosocial, lenguaje y adaptativo. Al final, se evaluó nuevamente el desarrollo con la misma escala. Resultados y discusión: La edad límite que obtuvo en la evaluación basal fue de 7 meses de edad. Después de la intervención se pudo observar una mejora en su control postural, en sus reacciones posturales y de equilibrio. La evolución del niño después del tratamiento fisioterapéutico fue favorable en el periodo evaluado, según los resultados obtenidos. La evaluación final con la Escala de Gesell dio una edad límite de 1 año 4 meses. Conclusión: El programa corto de fisioterapia aplicado al paciente mostró efectos favorables en su desarrollo psicomotriz.

\section{Palabras Clave:}

Parálisis cerebral, Discinética, control postural, fisioterapia.

\section{Introducción}

La parálisis cerebral infantil $(\mathrm{PCl})$ es un término que describe trastornos motores, del equilibrio y la postura

\footnotetext{
Autor de Correspondencia, Universidad Autónoma del Estado de Hidalgo, https://orcid.org/0000-0003-1047-6304, Email: mortiz@uaeh.edu.mx

b Universidad Autónoma del Estado de Hidalgo, https://orcid.org/0000-0001-7791-858X, Email: marlenneyuli@ gmail.com

• Cuerpo Académico Fisioterapia Multidisciplinaria, Universidad Politécnica de Pachuca, https://orcid.org/0000-0002-2218-8740, Email: kristalcortes@upp.edu.mx
} 
ocasionados por el daño cerebral. La $\mathrm{PCl}$ es la discapacidad motora más frecuente en la niñez, se estima que dos de cada 1,000 niños recién nacidos desarrollarán este tipo de parálisis. ${ }^{1-6}$ Las manifestaciones podrían cambiar en el transcurso de la vida de un niño a medida que su sistema nervioso madura. Esto significa que los síntomas podrían cambiar con el paso del tiempo. ${ }^{1}$ Según las áreas del cerebro afectadas, en la $\mathrm{PCl}$ puede presentarse rigidez muscular, movimientos involuntarios y falta de equilibrio y de coordinación. Los movimientos son inconscientes, y pueden ser lentos o rápidos, y espasmódicos. Las personas con este tipo de discapacidad tienen problemas para controlar los movimientos de sus manos, brazos, pies y piernas, lo que les dificulta estar sentadas y caminar. A veces se afectan la cara, la lengua, y la persona tiene dificultad para succionar, tragar y hablar. ${ }^{7-10}$ Además de afectar la motricidad y la postura del paciente, en la $\mathrm{PCl}$ se puede dañar la visión, la audición, el lenguaje y la inteligencia; es muy frecuente también que aparezcan episodios de epilepsia. $^{2-4}$

Hay cuatro tipos de $\mathrm{PCl}$ : espástica, discinética, atáxica y mixta. El tipo de $\mathrm{PCl}$ discinética tiene una frecuencia de 10 al $15 \% .^{2}$ En ésta puede haber variaciones durante el desarrollo, por ejemplo, hipotónico poco después del nacimiento, pero más adelante puede desarrollar espasticidad. ${ }^{2,3} \mathrm{La} \mathrm{PCl}$ discinética de tipo distónica (PCIDD) causa movimientos musculares lentos, involuntarios y bruscos que pueden ser repetitivos. ${ }^{2-4}$

Diversos estudios han demostrado las habilidades que puede logra un paciente con PCIDD después de recibir tratamiento fisioterapéutico específico. ${ }^{1-4}$ Como un primer paso, se debe evaluar el nivel de desarrollo de la conducta del paciente (motora, adaptativa, lenguaje, emocional, social) mediante la escala de desarrollo psicomotor de Gesell. Después de esto, se elabora un plan fisioterapéutico específico integral para ser aplicado de manera escalonada. En el transcurso de la fisioterapia, se va valorando de manera detallada los avances en el desarrollo del paciente de acuerdo a su edad. ${ }^{4,6,11,12}$ En este sentido, el objetivo de este reporte es describir el desarrollo psicomotor de un niño con PCIDD mediante un abordaje fisioterapéutico integral.

\section{Presentación del caso clínico}

Paciente masculino de 2 años de edad, diagnosticado con PCIDD, el cual acude a evaluación y tratamiento fisioterapéutico. Como antecedentes heredofamiliares, no se presenta ningún caso de este tipo de enfermedad, ni ningún tipo de enfermedades crónico-degenerativas en la familia por parte de la madre ni del padre. Los padres del paciente, siendo pareja, no utilizaron ningún método anticonceptivo. Al enterarse del embarazo, el padre se ausenta. La madre es hija única y con 25 años al momento del embarazo. La madre no presenta ninguna patología de importancia al momento de iniciar el embarazo. Es su primer embarazo. En sus antecedentes gineco-obstétricos no tuvo buena alimentación ni tomo ácido fólico durante el embarazo. Asimismo, refiere no haber usado medicamentos o drogas (licitas o ilícitas) durante el embarazo. Presentó una amenaza de aborto en el primer trimestre, debido a maltrato físico y psicológico por parte de la suegra con el fin de que abortara. No tuvo seguimiento médico prenatal. La madre inició con trabajo de parto pretérmino espontáneo (se desconoce la probable causa) a las 32 semanas de embarazo y acude al servicio de urgencias del Hospital General de Pachuca, Hidalgo. En el hospital la ingresan e inician tratamiento con líquidos y medicamentos útero-inhibidores (desconoce la madre qué medicamentos le administraron). En el hospital no logran inhibir la progresión del embarazo, por lo que pasó a la sala de expulsión y nació el niño por parto de 32 semanas. Al momento del parto, el niño presenta paro respiratorio y con signos neurológicos alterados, con Apgar de 1 (al minuto) y 6 (a los 5 minutos).

Después de su nacimiento, el niño permaneció en la Unidad de Cuidados Intensivos Neonatales del hospital. El paciente permaneció en el Hospital por 18 semanas y durante las cuales presentó diversas complicaciones (neumonía, sepsis y epilepsia). El paciente es dado de alta del hospital a los 3 meses. Después de que es dado de alta, el paciente es llevado de manera esporádica a fisioterapia al mismo hospital General. La madre refiere que llevó al paciente a sesiones de fisioterapia aproximadamente unas 10 veces (desde el cuarto mes al mes 23 de vida del paciente).

La madre en busca de obtener tratamiento con mejor y más rápido avance para su hijo, acude a consulta al Centro de Rehabilitación y Neurodesarrollo Infantil en la Ciudad de Pachuca, Hidalgo. El paciente es valorado por el Médico Especialista (al momento de su evaluación cuenta con 2 años de edad). Se realizó historia clínica y se valora su capacidad psicomotora. Se diagnostica al paciente con retraso severo psicomotor y parálisis cerebral infantil discinética de tipo distónica (PCIDD). Respecto a los hitos del desarrollo, el niño no mostró sostén cefálico, ni sedestación, no hubo control de tronco, rotaciones, giros y arrastre, había hipotonía generalizada, y estrabismo del ojo izquierdo. Se realizó una primera valoración con la Escala de Gesell, y se determinó una edad base del paciente de 7 meses (Tablas 1-3). Se realiza la programación junto con la madre, para que el paciente inicie con sus sesiones de fisioterapia.

\section{Intervención}

Partiendo de su edad base de 7 meses, se planificó un tratamiento fisioterapéutico integral..$^{2,4,6,11-14} \mathrm{El}$ programa 
de fisioterapia constó de ejercicios que siguen una progresión y enfocados en sus hitos de desarrollo y que tuvieron que realizarse 2 veces por semana durante 3 meses. Cada sesión con una duración de 60 minutos. También se tuvo terapia en grupo con otros niños.

Los materiales necesarios fueron: colchoneta, equipo de sonido con música para las sesiones, pelota Bobath, bandeja, cepillos, texturas, percutor pediátrico, semillas, burbujas, luces de colores, aceite y crema.

Posterior a los 3 meses de tratamiento, se realizó una evaluación final con la misma escala de Gesell y se determinó su edad límite.

\section{Descripción del Programa de Fisioterapia}

1. Como inicio de la terapia se preparan las articulaciones para el ejercicio, para fortalecer y no lastimar los músculos del paciente. En este sentido, se le coloca en decúbito supino, sobre la colchoneta, y se realiza la movilización de sus articulaciones, desde dedos, muñeca, codo, hombros, cadera, rodillas, tobillos. Series de tres movilizaciones en cada articulación, 10 repeticiones por cinco minutos en cada sesión. Hacerlo de manera muy lenta ya que por la hipotonía que presenta, puede causar daño o molestia.

2. Estimulación sensorial con texturas. El paciente fue colocado en sedestación sobre la colchoneta con una bandeja enfrente de él. Se colocaron en la bandeja diferentes materiales, de diferentes texturas, que el paciente tocaba y cogía, como arroz, frijol, lentejas, tierra, etc. Esto ayuda a estimular el sentido del tacto para así generar reacciones y atención del niño. Posteriormente se realizaba un cepillado en palmas de manos y pies. Con el percutor pediátrico se le daban pequeños golpes sobre los vientres musculares para generar respuestas de contracción y relajación, lo que causa estimulación de músculos tónicos del niño (Figura 1). Esta parte se otorgó por 15-20 minutos en cada sesión.

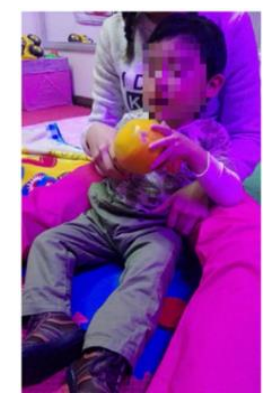

Figura 1. Paciente en sedestación sobre colchoneta con objeto estimulador.

3. El paciente se colocó en sedestación sobre la colchoneta y se realizaron coaptaciones articulares para estimular los tendones de músculos y fascias, partiendo de dedos, muñecas, codos, hombros, caderas, y rodillas. Se realizaron cinco repeticiones por cada articulación por
10-15 minutos en cada sesión. El objetivo de estas estimulaciones es mejorar sus respuestas a los movimientos y así generar coordinación y más control sobre sí mismo.

4. El paciente es colocado en posición de caballero (hincado) sobre la colchoneta (asistido por la terapeuta) para controlar la postura, la marcha en coordinación y mantener el equilibrio. Se realizaron 10 repeticiones, bilateral, para avanzar con ayuda de la pelota Bobath, para así llegar a la bipedestación (Figura 2).

5. Musicoterapia durante los 60 minutos que duró cada sesión de fisioterapia. Se eligió un tipo de música relajante o motivadora para generar un ambiente de confianza al niño, ocupando burbujas, luces de colores, espuma etc. Al final de cada sesión se otorgaba un masaje terapéutico con crema o aceite en miembros superiores e inferiores y la zona del abdomen. Lo anterior funciona para estimular los sentidos, ayudando a evitar de una terapia tediosa y dolorosa.

6. Se indicó una hora diaria de trabajo en casa, con ejercicios con pinzas, tarea en libreta de motricidad fina, trabajar paracaídas sobre pelota de Bobath y ejercicios de equilibrio para activar su reacción refleja.

\section{Evolución del paciente durante el tratamiento fisioterapéutico}

1. En las tablas 1-3 se observa que el paciente presentó un gran retraso en su desarrollo al inicio (al momento de admisión). En la esfera adaptativa solo sostenía objetos, golpeaba y los aventaba. Durante el primer mes de terapia fue muy difícil debido a que lloraba, y le dolían los ejercicios. Posteriormente se fue avanzando y logrando la sedestación, que tuviera la posición de gateo, y la bipedestación con ayuda.

2. Durante el segundo mes sus sentidos se empezaron activar y respuestas a los ejercicios, aumentó su atención y el trabajo de texturas ayudó. El agarre de manos con objetos aumentó, acomodando, sosteniéndolos, y lanzándolos, así ayudo a su pinza fina y logró también aplaudir. En su motricidad gruesa se mantenía en sedestación, solo presentaba control cefálico, y había presión palmar. En su lenguaje presentaba sonidos polisilábicos y reaccionaba ante los sonidos.

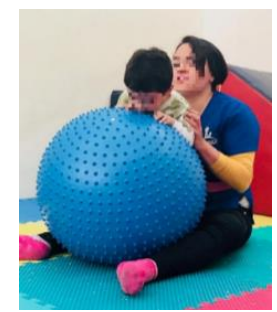

Figura 2. Paciente en bipedestación sobre la pelota Bobath. 
Tabla 1. Resultados de la evaluación con la Escala de Gesell al ingreso al programa fisioterapéutico.

\begin{tabular}{|c|c|c|c|c|}
\hline \multirow{2}{*}{\multicolumn{2}{|c|}{ ÁREAS }} & \multirow{2}{*}{ EDAD (7 MESES) } & \multicolumn{2}{|c|}{ Indicadores } \\
\hline & & & SI & NO \\
\hline \multirow{7}{*}{\multicolumn{2}{|c|}{ ADAPTATIVA }} & Con la sonaja y la campana, intenta acercarse y sujetar con una mano & $\mathrm{X}$ & \\
\hline & & Con los cubos es capaz de sostener uno y agarrar otro & $\mathrm{X}$ & \\
\hline & & Mantiene dos por un breve tiempo & $\mathrm{X}$ & \\
\hline & & Golpea la campana contra la mesa & $\mathrm{X}$ & \\
\hline & & Pasa de mano en mano cubos & $\mathrm{X}$ & \\
\hline & & Con la campana es capaz de detenerla y agarrarla. & $\mathrm{X}$ & \\
\hline & & Retiene la campana. & $\mathrm{X}$ & \\
\hline \multirow[t]{6}{*}{ MOTRIZ } & \multirow[t]{4}{*}{ GRUESA } & En posición supina levanta la cabeza & $\mathrm{X}$ & \\
\hline & & Sentado nuevamente, se mantiene inclinado hacia adelante apoyado sobre las manos & $\mathrm{X}$ & \\
\hline & & Se mantiene momentáneamente erecto & $\mathrm{X}$ & \\
\hline & & Parado sostiene gran parte del peso & $\mathrm{X}$ & \\
\hline & \multirow[t]{2}{*}{ FINA } & Hay presión palmar en cubos & $\mathrm{X}$ & \\
\hline & & Intenta sujetar la bola de estambre, con toda la mano mientras la toca. & $\mathrm{X}$ & \\
\hline \multirow{5}{*}{\multicolumn{2}{|c|}{$\begin{array}{l}\text { LENGUAJE } \\
\text { PERSONA SOCIAL }\end{array}$}} & Encuentra la voz, usa sonidos vocales polisilábicos y “m, m, m”, llora & $\mathrm{X}$ & \\
\hline & & Referente a los alimentos toma bien los sólidos. & $\mathrm{X}$ & \\
\hline & & En cuanto al juego lleva el pie a la boca. (En posición supina). & $\mathrm{X}$ & \\
\hline & & Es capaz de tocar el espejo y acariciar la imagen. & $\mathrm{X}$ & \\
\hline & & Se agita y abandona el esfuerzo. & $\mathrm{X}$ & \\
\hline
\end{tabular}

Tabla 2. Resultados de la evaluación con la Escala de Gesell a los 3 meses del programa fisioterapéutico.

\begin{tabular}{|c|c|c|c|c|}
\hline \multirow{2}{*}{\multicolumn{2}{|c|}{ ÁREAS }} & \multirow{2}{*}{ EDAD (1 AÑO 4 MESES) } & \multicolumn{2}{|c|}{ Indicadores } \\
\hline & & & SI & NO \\
\hline \multirow{6}{*}{\multicolumn{2}{|c|}{ ADAPTATIVA }} & Torre de 2 & $\mathrm{X}$ & \\
\hline & & Coloca 6 cubos & $\mathrm{X}$ & \\
\hline & & Incipiente imitación del trazo & $\mathrm{X}$ & \\
\hline & & Coloca bloque redondo & $\mathrm{X}$ & \\
\hline & & Tablero excavado: Adapta bloque & $\mathrm{X}$ & \\
\hline & & Coloca bloque redondo rápidamente & & $\mathrm{X}$ \\
\hline \multirow[t]{7}{*}{ MOTRIZ } & \multirow[t]{5}{*}{ GRUESA } & Pocos pasos, inicia, se para & $\mathrm{X}$ & \\
\hline & & Caer por derrumbe. & $\mathrm{X}$ & \\
\hline & & Abandona el gateo. & $\mathrm{X}$ & \\
\hline & & Sube gateando (escalera) & & $\mathrm{X}$ \\
\hline & & Torre de 2 & $\mathrm{X}$ & \\
\hline & \multirow[t]{2}{*}{ FINA } & Introduce bolitas en la botella (sin demostración). & $\mathrm{X}$ & \\
\hline & & Ayuda a volver páginas. & $\mathrm{X}$ & \\
\hline \multirow{4}{*}{\multicolumn{2}{|c|}{ LENGUAJE }} & 4 o 5 palabras, incluyendo nombres & & $\mathrm{X}$ \\
\hline & & Usa jerga & $\mathrm{X}$ & \\
\hline & & Acaricia dibujos & $\mathrm{X}$ & \\
\hline & & Dice "da-da" o equivalentes. & $\mathrm{X}$ & \\
\hline \multirow{8}{*}{\multicolumn{2}{|c|}{ PERSONA SOCIAL }} & Inhibe agarrar plato. & $\mathrm{X}$ & \\
\hline & & Control del esfínter: Regularización parcial. & & $\mathrm{X}$ \\
\hline & & Control rectal. & & $\mathrm{X}$ \\
\hline & & Indica ropa mojada. & $\mathrm{X}$ & \\
\hline & & Descarta biberón. & $\mathrm{X}$ & \\
\hline & & Señala y parlotea, cuando desea algo. & $\mathrm{X}$ & \\
\hline & & En el juego muestra o enseña juguetes. & $\mathrm{X}$ & \\
\hline & & En el juego arroja juguetes durante el juego o como rechazo. & $\mathrm{X}$ & \\
\hline
\end{tabular}

\section{Discusión}

Se presenta caso clínico de paciente de 2 año de edad a su admisión y con diagnóstico de PCIDD. De acuerdo a la Escala de Gesell, el paciente presentaba una edad base de 7 meses al momento de iniciar el programa de fisioterapia. Se inicia terapia fisioterapéutica integral en el Centro de Rehabilitación y con ejercicios en casa. Después de 3 meses de terapia, se observa una buena evolución del paciente y se realiza una nueva valoración de Gesell, obteniendo una edad límite de 1 año 4 meses. Se considera que la estimulación y los ejercicios se debe realizar de manera constante, sin perder seguimiento de su tratamiento, ya que el niño puede atrasarse aún más en su proceso de rehabilitación. Cada actividad realizada durante su fisioterapia es un logro y con esto va superando su desarrollo psicomotriz y ofrece un pronóstico de vida mejor del niño.

En un reporte clínico, se aplicó el concepto Bobath 5 veces por semana, por 45 min cada sesión, por 16 semanas a un niño de 9 años con diagnóstico de parálisis cerebral de tipo cuadriplejia espástica nivel V. Los resultados obtenidos mostraron que 
el concepto Bobath genera cambios positivos significativos en la función motora y el manejo de la espasticidad. ${ }^{13}$ Lo cual apoya los avances de nuestro paciente en la utilización del concepto Bobath.

Tabla 3. Resultados de la evaluación del Desarrollo Psicomotor (Escala de Gesell)

\begin{tabular}{|c|c|c|}
\hline $\begin{array}{l}\text { Evaluación } \\
\text { inicial }\end{array}$ & Indicadores & Resultado inicial \\
\hline Adaptativa & $7-7$ & \multirow{5}{*}{$\begin{array}{l}\text { Según los ítems, el } \\
\text { paciente obtuvo una edad } \\
\text { base de } 7 \text { meses } 028 \\
\text { semanas. }\end{array}$} \\
\hline Motriz gruesa & $3-3$ & \\
\hline Motriz fina & $3-3$ & \\
\hline Lenguaje & $1-1$ & \\
\hline Psicosocial & 4-4 & \\
\hline $\begin{array}{l}\text { Evaluación } \\
\text { Final }\end{array}$ & Indicadores & Resultado Final \\
\hline Adaptativa & $4-5$ & Después del tratamiento \\
\hline $\begin{array}{l}\text { Motriz } \\
\text { gruesa }\end{array}$ & $3-4$ & $\begin{array}{l}\text { fisioterapéutico se obtuvo } \\
\text { un avance en sus }\end{array}$ \\
\hline Motriz fina & $3-3$ & indicadores, obteniendo \\
\hline Lenguaje & $2-3$ & una edad límite de 1 año 4 \\
\hline Psicosocial & $7-9$ & meses o 16 meses. \\
\hline
\end{tabular}

En otro caso clínico, se evaluó la efectividad de un programa fisioterapéutico integral (como el nuestro) a una paciente con retraso del desarrollo psicomotor y hemiparesia izquierda. Los resultados evidenciaron una mejora importante en el tono muscular y aumento significativo de la movilidad en el miembro superior izquierdo, así como una realización autónoma de los cambios posturales más importantes. ${ }^{14}$ Lo anterior apoya la efectividad de los programas fisioterápicos integrales, como en nuestra paciente.

\section{Conclusión}

La evolución del niño después del tratamiento fisioterapéutico integral fue favorable en un periodo corto. Con ayuda multidisciplinaria y apoyo de la madre, se lograron diferencias significativas, aún con el tipo de parálisis presentado.

\section{Responsabilidades éticas}

Derecho a la privacidad y consentimiento informado. Los autores han obtenido el consentimiento informado de los pacientes y/o sujetos referidos en el artículo.

\section{Conflicto de interés}

Los autores declaran no tener ningún conflicto de intereses.

\section{Financiación}

La presente investigación no ha recibido ayudas específicas provenientes de agencias del sector público, sector comercial o entidades sin ánimo de lucro.

\section{Referencias}

[1] Palisano R, Rosenbaum P, Walter S, Russell D, Wood E, Galuppi B. Development and reliability of a system to classify gross motor function in children with cerebral palsy. Dev Med Child Neurol. 1997;39:214-23. https://doi.org/10.1111/j.1469-8749.1997.tb07414.x

[2] Guía de Práctica Clínica Abordaje y Manejo del Niño con Parálisis Cerebral Infantil con Comorbilidades Neurológicas y Músculo Esqueléticas. México: Secretaría de Salud; 2010. http://www.cenetec.salud.gob.mx/interior/gpc.html (Consultada el 25 de marzo del 2020).

[3] Hay Jr WW, Levin MJ, Deterding RR, Abzug MJ. Current Diagnosis and Treatment Pediatrics. 25th ed. Lange; 2018.

[4] Shelov SP, Hannemann RE. Caring for your baby and young child: Birth to age 5. 2th ed. American Academy of Pediatrics; 2005.

[5] Vázquez Vela CC, Vidal Ruiz CA. Parálisis cerebral infantil: definición y clasificación a través de la historia. Rev Mex Ortop Ped. 2014;16:6-10

[6] Lois Guerra J. Manual de fisioterapia. 2th Ed. México: El Manual modern; 2018

[7] Rizzoli-Córdoba A, Campos-Maldonado MC, Vélez-Andrade VH, Delgado-Ginebra I, Baqueiro-Hernández CI, Villasís-Keever MA, et al. Evaluación diagnóstica del nivel de desarrollo en niños identificados con riesgo de retraso mediante la prueba de Evaluación del Desarrollo Infantil. Bol Med Hosp Infant Mex. 2015;72:397-408. https://doi.org/10.1016/j.bmhimx.2015.11.005

[8] Comisión Nacional de Protección Social en Salud. Manual para la evaluación de menores de cinco años con riesgo de retraso en el desarrollo. Primera edición. México D.F.: Secretaría de Salud, 2013. http://himfg.com.mx/descargas/documentos/EDI/ManualparaEvaluaci onaMenoresde5conRiesgodeRetrasoenelDesarrollo.pdf (Consultada el 31 de julio del 2002).

[9] Robaina Castellanos GR. Asociación entre factores perinatales y neonatales de riesgo y parálisis cerebral. Rev Cubana Pediatr 2010;82. http://scielo.sld.cu/scielo.php?script=sci_arttext\&pid=S003475312010000200008 (Consultada el 31 de julio del 2002).

[10]Fejerman N, Fernández Álvarez E. Neurología Pediátrica. $3^{\text {a }}$ ed. España: Editorial Médica Panamericana; 2007.

[11]Gonzales Arévalo MP. Fisioterapia en Neurología: Estrategias de intervención en Parálisis cerebral. Umbral Científico 2008;7:24-32.

[12] Morante R MM, Lillo SS, Cubillos LA. Impacto de las nuevas terapias en el manejo de la hipertonía en el niño con parálisis cerebral. Rev Med Clin Condes 2014;25:315-29. https://doi.org/10.1016/S07168640(14)70043-4

[13]Lerma Castaño P. R, Chanaga Gelves M.V, Perdomo Urazan D. Abordaje de un caso de parálisis cerebral espástica nivel v mediante el concepto Bobath. Fisioterapia 2019;41:242-6. https://doi.org/10.1016/j.ft.2019.03.006.

[14]Bueno Martín A, Domínguez Oliván MP. Tratamiento fisioterápico mediante el concepto Bobath de un caso de hemiparesia por parálisis cerebral infantil. Universidad de Zaragoza, Facultad de Ciencias de la Salud. 2013 https://zaguan.unizar.es/record/10929?ln=es\# 